\title{
Transition of the mushy zone from continuous liquid films to a coherent solid
}

\author{
S. Vernède and M. Rappaz \\ Computational Materials Laboratory, Ecole Polytechnique Fédérale de Lausanne \\ MXG, Station No 12, CH-1015 Lausanne, Switzerland \\ Tel: +41-21-693 2844 - E-mail: michel.rappaz@epfl.ch, stephane.vernede@epfl.ch
}

Keywords : solidification, hot tearing, coalescence, coherency

Published in Philosophical Magazine, Vol. 86, No. 24, 21 August 2006, 3779-3794

view at publisher

\begin{abstract}
While studies of solidification microstructures have focused mainly on the tips of the dendrites, the last stage solidification is equally important from the point of view of defect formation (porosity, hot tearing), mechanical strength build-up and precipitation of phases. In particular, the transition from continuous liquid films to a coherent solid in low concentration alloys is of crucial importance for hot tearing formation, and more generally speaking for liquid feeding ability and coherency development. Based on a fairly recent theoretical model of coalescence which will be recalled briefly, new results obtained for a population of equiaxed grains will be presented. A granular-type model based on a Voronoi tessellation has been used for the description of the gradual disappearance of liquid films and the clustering of equiaxed grains. This percolation-type approach has been used then to calculate the pressure drop in the mushy zone on the assumptions of a Poiseuille flow in between the grains and a Kirchhoff model for the connectivity of the liquid films including the Losses associated with solidification shrinkage (i.e, PKL model). Comparison with a standard average pressure drop calculation based on Carman-Kozeny’s relationship will be presented.
\end{abstract}




\section{Introduction}

Hot tearing, together with microporosity, is one of the most severe defects occurring during casting of low-concentration metallic alloys. It has been the subject of numerous studies, which are very well summarized in the recent review paper of Eskin et al [1]. From the numerous experimental and simulation studies on this topic, it appears that hot tears form at grain boundaries as a result of combined tensile/shear stresses induced by the coherent solid and insufficient interdendritic liquid feeding. The hot cracking sensitivity is most acute for low concentration alloys for which thin liquid films still exist at grain boundaries up to very high volume fraction of solid, typically 0.9 to 0.95 . Scanning electron microscopy observations of hot tear surfaces revealed fairly smooth surfaces made of secondary dendrite arms stopped during their growth and not yet "linked" to arms on the opposite side of the grain boundary. The few spikes observed on these surfaces were the result of either the last solidification stage of liquid menisci or the stretching of solid bridges already established across the grain boundary before deformation of the mushy zone. Such conclusions have been evidenced by direct in-situ observations performed on organic substances [2].

The last stage solidification in which a continuous liquid film network is gradually replaced by a coherent solid is therefore of primary importance to the understanding and modeling of hot tearing formation. Indeed, recent two-phase models developed for hot tearing are based on either a unique critical temperature, $T_{c}$, at which solid coherency is reached $[3,4]$, or an internal variable of a rheological model describing the state of coalescence of the solid $[5,6]$. In high concentration alloys, this transition is achieved by the formation of interdendritic eutectic whereas, at lower concentrations, it is made through numerous coalescence events. By coalescence, we mean in the present contribution the disappearance of a liquid film in between two distinct grains (or two dendrites arms of the same grain), leading to the formation of a solid grain boundary (or of a single grain) with a few isolated pockets of liquid.

The problem of coalescence, the opposite of grain boundary wetting, during solidification has been tackled recently by Rappaz et al [7] using a sharp interface approach and a multi-phase field method. The first method is interesting for simple 1-dimensional (1D) situations while the second one allows the treatment of realistic grain boundary morphology, but at a very high computation cost. An interesting alternative approach has been proposed by Mathier et al 
[8] : it considers an ensemble of equiaxed grains nucleating at the same time and growing at the same speed, thus resulting in a final grain boundary network made of linear segments (i.e., corresponding to the Voronoi tessellation associated with the nucleation centers).

In the present contribution, the model of Mathier et al is further simplified and extended in order first, to treat a very large number of grains at a reasonable computation cost while retaining the basic physical mechanisms, and second, to account for the non-uniform temperature field of an entire mushy region. This part is presented in section 2. This model is then coupled to an interdendritic liquid flow model similar to that described by Dijkstra et al [9], but with a stochastically random network of grains. The pressure drop induced by solidification shrinkage in this liquid film network is compared with a standard Darcy-type approach of liquid feeding. After presenting this model in Section 3, a few results are given in Section 4, while Section 5 briefly outlines future work based on this "granular approach”.

\section{A granular model of solidification and coalescence}

Using a sharp interface approach, Rappaz et al have analyzed coalescence from a thermodynamic point of view [7]. Considering two planar solid-liquid interfaces which meet at the future grain boundary, they showed that coalescence within a pure substance will occur at an undercooling, $\Delta \mathrm{T}_{\mathrm{b}}$, below the freezing point :

$$
\Delta \mathrm{T}_{\mathrm{b}}=\frac{\gamma_{\mathrm{gb}}-2 \gamma_{\mathrm{sl}}}{\Delta \mathrm{s}_{\mathrm{f}}} \frac{1}{\delta}
$$

where $\Delta \mathrm{s}_{\mathrm{f}}$ is the entropy of fusion per unit volume, $\delta$ is the thickness of the diffuse interface and $\gamma_{\mathrm{gb}}$ and $\gamma_{\mathrm{sl}}$ are the interfacial energies of the (dry) grain boundary and the solid-liquid interface, respectively. Such an approach has been substantiated recently by molecular dynamics calculations [10]. From this expression, three situations have to be considered. When $\gamma_{\mathrm{gb}}<2 \gamma_{\mathrm{s} / 1}$ (i.e., $\Delta \mathrm{T}_{\mathrm{b}}<0$ ), coalescence is associated with a decrease in free energy and will occur as soon as the two solid-liquid interfaces impinge. This attractive situation is typical of dendrites belonging to the same grain $\left(\gamma_{\mathrm{gb}}=0\right)$ or on each side of a low-angle grain boundary. When $\gamma_{\mathrm{gb}}=2 \gamma_{\mathrm{s} / 1}$ (i.e., $\Delta \mathrm{T}_{\mathrm{b}}=0$ ), there is no surface energy gain during coalescence

and the case is referred to as a neutral grain boundary. When $\gamma_{g b}>2 \gamma_{s / l}$ (i.e., $\Delta T_{b}>0$ ), a 
driving force is required for bridging since the interfacial energy balance is not favorable. This repulsive situation is typical of high-angle grain boundaries.

For binary alloys, the situation is similar providing the undercooling $\Delta \mathrm{T}_{\mathrm{b}}$ is replaced by a coalescence line (surface in multicomponent alloys) : this coalescence line is parallel to, but $\Delta \mathrm{T}_{\mathrm{b}}$ below, the liquidus. Indeed, in alloys, back-diffusion perpendicular to the impinging interfaces and diffusion in the liquid parallel to the film can contribute to coalescence. In order to handle such situations in 1D, Rappaz et al coupled this sharp interface coalescence model with a 1D back-diffusion calculation [7]. Mathier et al extended this approach to a network of grains, as shown in Fig. 1 [8]. Assuming globulitic grains, which are typical of heavily inoculated alloys, these authors generated $\mathrm{N}$ nuclei of random location and orientation. With the assumption that the grains form at the same time and grow with the same speed, the final grain boundaries are straight segments corresponding to the Voronoi tessellation of the nucleation centers [11].This Voronoi tessellation is generated using the free-access software Qhull [12]. In order to further simplify the solidification model, the smooth interface of each grain during growth was approximated by a linear segment in each triangle connecting the nucleation center with a Voronoi segment (Fig. 1b). By construction, these segments are perpendicular to the vectors connecting the nucleation centers. The advance of each solid-liquid interface segment is calculated during solidification with the help of a microsegregation model [8]. When an interface segment is at a distance $h$ from the final grain boundary, its symmetrical counterpart is at the same distance, i.e., the liquid film has a thickness $2 \mathrm{~h}$ (Fig. 1b). The orientation of each grain, $\theta_{\mathrm{I}}$, is defined randomly (i.e., from 0 to 45 deg. in 2D with a 4-fold symmetry of the structure) and the grain boundary energy between two grains, $\gamma_{\mathrm{gb}, \mathrm{IJ}}$, is calculated in the same way as Mathier et al, i.e., assuming a simplified Read-Shockley distribution $\gamma_{\mathrm{gb}}\left(\theta_{\mathrm{I}}-\theta_{\mathrm{J}}\right)$.

Diffusion perpendicular to the solid-liquid interface segments was calculated by Mathier et al in both the solid and liquid phases until coalescence was terminated. As the grain size was on the order of $100 \mu \mathrm{m}$ while the diffuse interface thickness $\delta$ is only a few $\mathrm{nm}$, such microsegregation calculations was very computation intensive. In the present calculations, diffusion in the liquid phase was assumed to be complete. Furthermore, when the thickness (2h) of the liquid layer remaining in between two grains is on the order of $\delta$, the advance of the interface is stopped until the coalescence line is reached, point at which the liquid film 
disappears abruptly. Back-diffusion in the solid was calculated according to a Landau transformation, which applies the solid segment $\left[0, \mathrm{x}^{*}(\mathrm{t})\right]$ onto a reference $1 \mathrm{D}$ domain $[0,1]$ [13]. The model is therefore similar to that of Ohnaka [14] and will not be described here. Finally, the uniform temperature assumption of Mathier et al is replaced by a simple thermal history typical of a Bridgman furnace, i.e., $T(z, t)=T_{o}+G\left(z-v_{T} t\right)$, where $T_{o}$ is a reference temperature, $\mathrm{G}$ is the thermal gradient along the $\mathrm{z}$-direction and $\mathrm{v}_{\mathrm{T}}$ the speed of the isotherms.

\section{A discrete model of interdendritic flow}

Since the density of the solid, $\rho_{\mathrm{s}}$, is not equal to that of the liquid, $\rho_{\mathrm{l}}$, the advance of the interface in each triangle of the tesselation induces some intergranular liquid flow (Fig. 2a). Assuming that $\rho_{\mathrm{l}}$ and $\rho_{\mathrm{s}}$ are constant and that the solid is fixed, the liquid flow at the solidliquid interface has a velocity $\mathrm{v}_{\mathrm{l}, \mathrm{n}}$ given by:

$$
\mathrm{v}_{\mathrm{l}, \mathrm{n}}=-\beta \mathrm{v}^{*}
$$

where $\beta$ is the solidification shrinkage, i.e., $\beta=\left(\rho_{s} / \rho_{l}-1\right)$, and $v^{*}$ is the speed of the moving interface. The normal $\mathbf{n}$ to the interface along which $\mathbf{v}_{\mathbf{l}}$ is projected is pointing toward the liquid. Assuming that no pore forms, the mass conservation equation for the incompressible liquid domain is simply: $\operatorname{div} \mathbf{v}_{\mathbf{l}}=0$. This equation can be integrated over the trapezoidal liquid volume (surface in 2D) remaining within each triangle (see Fig. 2), taking into account the symmetry of the flow with respect to the grain boundary. Taking now the normal pointing outward of the liquid domain and using the divergence theorem, one has:

$$
\int_{0}^{h} \operatorname{vl}, \mathrm{n} d x+\int_{0}^{h} \mathrm{vl,n} d x+\beta v^{*} S^{*}=0
$$

where $\mathrm{v}_{\mathrm{l}, \mathrm{n}}$ is the normal velocity in the liquid channel at the two extremities of the triangle, $\mathrm{S}^{*}$ is the surface (length in 2D) of the solid-liquid interface segment. The signed flow of liquid, $\mathrm{I}_{\frac{1}{2}, \text { in }}$, coming in over half the liquid channel width (first term in Eq. (3)) is negative, whereas that going out, $\mathrm{I}_{\frac{1}{2}, \text { out }}$, (second term in Eq. (3)) as well as the flow which compensates shrinkage over the linear interfacial segment are both positives. Equation (3) can therefore be written as: 


$$
\left|\mathrm{I}_{1 / 2, \text { in }}\right|-\left|\mathrm{I}_{1 / 2, \text { out }}\right|=\int_{0}^{\mathrm{h}}|\mathrm{vl}, \mathrm{in}| \mathrm{dx}-\int_{0}^{\mathrm{h}}|\mathrm{Vl}, \mathrm{n}| \mathrm{dx}=\beta \mathrm{v}^{*} \mathrm{~S}^{*}
$$

Since the shrinkage term is positive, this means that the exit flow, $\left|I_{1 / 2, \text { out }}\right|$, is smaller than the input flow, $\left|\mathrm{I}_{1 / 2, \text { in }}\right|$, in absolute values.

In order to simplify the equations, we define an s-axis parallel to the grain boundary and in the direction of the flow, i.e., the components of the velocity along this axis have the same (positive) sign at the entrance and exit of the channel. Assuming a Poiseuille flow at any location, s, of the liquid film (see Fig. 2), the velocity profile can be deduced from the maximum velocity at the center of the channel, $v_{\max }(\mathrm{s})$, or from the flow rate. The liquid flow within half a channel is indeed given by (the index " $n$ " appearing in $\mathrm{v}_{l}$ has been omitted for simplicity):

$$
I_{1 / 2}(s)=\int_{0}^{h} v_{l}(s, x) d x=v_{\max }(s) \int_{0}^{h}\left(1-\frac{x^{2}}{h^{2}}\right) d x=\frac{2 v_{\max }(s) h}{3}
$$

On the other hand, the Poiseuille flow within each channel is directly linked with the pressure gradient through the relationship:

$$
\mathrm{v}_{\max }(\mathrm{s})=-\frac{\mathrm{h}^{2}}{2 \mu} \frac{\mathrm{dp}}{\mathrm{ds}}
$$

Therefore, the flow within each half-channel is given by :

$$
\mathrm{I}_{1 / 2}(\mathrm{~s})=\frac{2 \mathrm{v}_{\max }(\mathrm{s}) \mathrm{h}}{3}=-\frac{\mathrm{h}^{3}}{3 \mu} \frac{\mathrm{dp}}{\mathrm{ds}}
$$

Equation (4), which corresponds to the integration of the mass balance over the entire length, $\mathrm{S}^{*}$, of the solid-liquid interface can also be viewed in a local form as:

$$
-\frac{\mathrm{dI}_{1 / 2}}{\mathrm{ds}}=-\frac{2 \mathrm{~h}}{3} \frac{\mathrm{dv}_{\max }}{\mathrm{ds}}=\frac{\mathrm{h}^{3}}{3 \mu} \frac{\mathrm{d}^{2} \mathrm{p}}{\mathrm{ds}^{2}}=\beta \mathrm{v}^{*}
$$

This equation is formally similar to the Darcy equation describing the flow in a porous medium when it is combined with the mass conservation equation for a solidifying medium. It can be easily extended to 3 dimensions for which the Voronoi tessellation will produce tetrahedral elements with triangular solid-liquid interfaces or grain boundaries. Over each of this triangular grain boundary, one has:

$$
\frac{\mathrm{h}^{3}}{3 \mu} \Delta \mathrm{p}=\frac{\mathrm{h}^{3}}{3 \mu}\left(\frac{\mathrm{d}^{2} \mathrm{p}}{\mathrm{ds}^{2}}+\frac{\mathrm{d}^{2} \mathrm{p}}{\mathrm{dt}^{2}}\right)=\beta \mathrm{v}^{*}
$$


where the Laplacian of the pressure field is taken along two axes, s and t, parallel to the grain boundaries.

It might look surprising to use a Poiseuille flow approximation (no-slip condition at the wall, stationary profile, flow parallel to flat and parallel boundaries, no gravity) for two reasons: first, at the beginning of solidification, the network of grains cannot be assimilated to a network of flat channels; second, deeper in the mushy zone, there is a flow normal to the solid-liquid boundary to compensate shrinkage. This approximation is nevertheless used in the present work for several reasons: first, it avoids using an intensive computation (as well complex enmeshment) of the real (2D) flow within the network; second, the pressure drop near the liquidus is nearly nil and of no interest for the purpose of studying hot tearing; third, a simple estimation of the magnitude of the parallel and normal components of the velocity field deeper in the mushy zone (where the pressure drop becomes significant) can be made. Assuming that a channel of width (2h) feeds $\mathrm{N}$ channels downwards the mushy zone (i.e., towards the solidus), the average parallel component of the velocity in this channel, $\left\langle\mathrm{v}_{\mathrm{l} / /}\right\rangle$, is equal to:

$$
\left\langle\mathrm{v}_{\mathrm{l} / /}>=\mathrm{v}_{\perp \perp} \frac{\mathrm{NL}}{\mathrm{h}}\right.
$$

where $L$ is the length of the channels (assumed all equal) and $v_{\perp \perp}$ is the normal component of the flow (given by $\beta \mathrm{v}^{*}$, see Eq. 2). Taking $\mathrm{L}=100 \mu \mathrm{m}$ and $\mathrm{h}=5 \mu \mathrm{m}$ (i.e., $\mathrm{g}_{\mathrm{s}}$ on the order of 0.9 ), this means that the ratio $\mathrm{v}_{\perp \perp} / \mathrm{V}_{\mathrm{l} / /}>$ is only $5 \%$, divided by the number of channels $\mathrm{N}$. This is therefore very close to a no-slip condition at the solid-liquid interface. By the same arguments, the pressure drop along a given channel is close to a linear approximation, despite the variation of the flow associated with feeding (Eq. 9).

Whether written in an integral or differential form, the present model is similar to a network of electrical resistances, since the velocity (current) is locally proportional to the pressure (voltage) gradient (i.e., the Poiseuille flow is similar to Ohm's law). However, this network has flow losses corresponding to the shrinkage term., i.e., the resistances are not well insulated from the board on which they are fixed. As any network, one must have a conservation of current/flow at any connecting node (Kirchhoff's law) (see Fig. 2b): 


$$
\sum_{v=1}^{6} I_{1 / 2, v}=0 \quad \text { at any nodal point }
$$

where the summation is made over all the half-channels connected to this node (6 for a regular triple junction of grain boundaries), the flows being signed with respect to the node.

This Kirchhoff-Poiseuille model which includes Losses is called KPL-model. For 2D or 3D geometry, it includes a Poiseuille flow within each linear segment/triangle of the solid-liquid interface of the grains, a Kirchhoff connection rule of all these channels and losses to compensate for shrinkage. Figure 3 shows such an overall model for a regular arrangement of hexagonal grains. From the solidification model (section 2 ) which gives the velocity $\mathrm{v}^{*}$ of all the interface segments/surfaces as well as their half-width, $h$, the sink term for the fluid flow is deduced (RHS term of Eq. (8) or (9)). The second-order partial differential equations giving the pressure $\mathrm{p}$ at all the nodes can then be solved using a weak Finite Element (FEM) formulation. In $2 \mathrm{D} / 3 \mathrm{D}$, the pressure problem is $1 \mathrm{D} / 2 \mathrm{D}$, i.e., the pressure to be found at the extremities of each grain boundary segment/triangle is expressed with linear hat/pyramid functions. The only difference with respect to standard 1D or 2D FEM simulations is the connectivity of the nodes. For example, in standard 1D problems, each node is only connected to two neighbors and the final matrix is tri-diagonal. In the present situation, most nodes are connected to 3 neighbors and the final matrix is sparse, with 4 non-zero terms on each line. Details of the resolution algorithm will be given elsewhere [15]. The boundary conditions of the problem are : a pressure imposed to the metallostatic pressure near the liquidus (triangles in Fig. 3) and a zero velocity on the last points to solidify (dots in Fig. 3), which is equivalent to a homogeneous Neumann condition for the pressure field.

\section{Results}

Figure 4 shows the type of result that can be obtained under stationary conditions for the directional solidification of an $\mathrm{Al}-1 \mathrm{wt} \% \mathrm{Cu}$ alloy. The thermal conditions of this Bridgmantype solidification are a vertical thermal gradient of $10^{4} \mathrm{~K} / \mathrm{m}$ and a velocity of $10^{-4} \mathrm{~m} / \mathrm{s}$ (i.e., cooling rate of $1 \mathrm{~K} / \mathrm{s}$ ). The average grain density is fixed to $10^{8} \mathrm{~m}^{-2}$, i.e., average grain size of $100 \mu \mathrm{m}$. There are 7200 grains in the present simulation to make them visible, but the model can handle a much larger number of grains within reasonable computation time. The 
temperature profile is shown on the right of Fig. 4 together with the average solid fraction profile estimated in horizontal sections of the grain structure shown at the center. The liquid is shown in black and the grains with various grey levels. As the grain is much smaller than the extent of the mushy zone (100 $\mu \mathrm{m}$ compared to more than $1 \mathrm{~cm})$, three enlargements of the grain structure are shown on the left with the corresponding scale. The location of these zones in the grain structure is indicated with white rectangles.

As can be expected from any microsegregation model, such low-concentration alloys are characterized by a primary phase which forms at a very early stage of solidification, i.e., near the liquidus. Therefore, the liquid channels are already fairly narrow in the enlargement located just below the liquidus (top of Fig. 4). At this solidification stage, their width is mainly a function of the distance separating the associated nucleation centers: the more distant the nucleation centers, the wider the liquid channels. Such findings are in agreement with more sophisticated phase field or pseudo-front tracking simulations [16]. As the temperature is further lowered, the liquid channels become thinner and the smaller ones have already disappeared (enlargement in the middle). At this stage, coalescence of the grains, and thus formation of clusters, is influenced by the grain boundary energy (Eq. (1)). However, a simpler criterion was used in the present case to determine coalescence: if the liquid channel becomes thinner than $1 \mathrm{~nm}$, it is closed. The reason of this choice is that a $1 \mathrm{~nm}$ liquid channel, i.e., on the order of the diffuse interface thickness, induces problems in the KPL calculation as the flow in such a channel becomes negligible. Close to the eutectic temperature (enlargement at the bottom), clusters made of several grains can be clearly identified since they are colored with the same grey level. Some liquid channels still remain within some of the clusters, but the volume fraction of the remaining liquid is very low (typically 1\%) when the eutectic reaction occurs (dashed line on the grain structure and on the temperature and volume fraction of solid curves on the right). Since no eutectic undercooling was considered in the present study, a single cluster is formed below (light grey).

Except for the non-uniform temperature field used in such simulations, the result of Fig. 4 is not different from that already obtained by Mathier et al [8]. However, the interest of such simulation is to provide a mean of calculating the pressure drop within such a network of channels (KPL model). Before presenting the results of such a calculation, the evolution of the effective solid-liquid interfacial area, $S_{s}$, is first shown in Fig. 5. It was calculated for a 
small volume element of uniform temperature. According to the notation of Nielsen et al [17], $\mathrm{S}_{\mathrm{s}}$ is given by:

$$
\mathrm{S}_{\mathrm{s}}=\frac{\mathrm{S}_{\mathrm{sl}}}{\mathrm{V}_{\mathrm{o}}}
$$

where $\mathrm{S}_{\mathrm{sl}}$ is the surface of the solid-liquid interface measured in a given volume (area in 2D), $\mathrm{V}_{\mathrm{o}}$. All these entities can be deduced from the granular solidification model as a function of the distance to the liquidus (Fig. 4) or as a function of the volume fraction of solid, $\mathrm{g}_{s}$. In order to be dimensionless, $\mathrm{S}_{\mathrm{s}}$ (unit $\mathrm{m}^{-1}$ ) has been normalized with the average grain size $\left(\mathrm{V}_{\text {tot }} / \mathrm{N}\right)^{1 / 2}$, where $\mathrm{V}_{\text {tot }}$ is the total volume (area) of the domain and $\mathrm{N}$ the total number of nuclei. As can be seen in Fig. 5, a regular hexagonal network of grains produces a monotonic evolution, $\mathrm{S}_{\mathrm{s}}\left(\mathrm{g}_{\mathrm{s}}\right)$, which follows the analytical expression:

$$
\mathrm{S}_{\mathrm{s}} \sqrt{\frac{\mathrm{V}_{\text {tot }}}{\mathrm{N}}}=2 \sqrt{2 \sqrt{3}} \sqrt{\mathrm{g}_{\mathrm{s}}}=2 \sqrt[4]{12} \sqrt{\mathrm{g}_{\mathrm{s}}}
$$

On the contrary, a random network of grains such as that shown in Fig. 4 produces a curve $\mathrm{S}_{\mathrm{s}}\left(\mathrm{g}_{\mathrm{s}}\right)$ which increases up to $\mathrm{g}_{\mathrm{s}}=0.95$, but then decreases to zero when $\mathrm{g}_{\mathrm{s}}$ tends toward unity. This can be understood simply based on geometric factors, regardless of coalescence. As a matter of fact, the curve shown in Fig. 5 was obtained without any consideration of the coalescence undercooling (Eq. (1)). Due to the random location of the nuclei, some grains are nucleating close to each other, whereas others are fairly far apart. In the first case, the boundary between the two grains is "closed” (i.e., solid) at a very early stage of solidification, whereas in the second case, it will remain “open” (i.e., liquid) up to a very late stage. Surprisingly, the critical value, $\mathrm{g}_{\mathrm{s}}=0.95$, beyond which the effective solid-liquid area decreases is very close to that mentioned in many hot tearing criteria for the point of coherency! Further investigations, especially 3D calculations, have to be performed in order to see whether this is just a fortuitous coincidence or not.

Having calculated the effective solid-liquid interfacial area from the granular model of solidification, the permeability, $\mathrm{K}$, of a packed bed can be estimated from the Carman-Kozeny relationship [17]:

$$
K=\frac{\left(1-g_{s}\right)^{3}}{5 S_{s}^{2}}=\frac{\left(1-g_{s}\right)^{3}}{5 S_{v}^{2} g_{s}^{2}}
$$

where $S_{V}$ is the intrinsic effective solid-liquid area normalized by the volume of solid, $V_{\mathrm{s}}$, instead of the volume $\mathrm{V}_{\mathrm{o}}$ : 


$$
\mathrm{S}_{\mathrm{V}}=\frac{\mathrm{S}_{\mathrm{sl}}}{\mathrm{V}_{\mathrm{s}}}=\frac{\mathrm{S}_{\mathrm{sl}}}{\mathrm{g}_{\mathrm{s}} \mathrm{V}_{\mathrm{o}}}=\frac{\mathrm{S}_{\mathrm{s}}}{\mathrm{g}_{\mathrm{s}}}
$$

Beyond the critical value $g_{s}=0.95$, the effective solid-liquid area of the random network decreases because thinner channels become closed, while all the channels of the regular network remain open. Beyond $\mathrm{g}_{\mathrm{s}} \approx 0.97$, the value of $\mathrm{S}_{\mathrm{s}}$ of the random network falls below that of the hexagonal network (while using the same density of grains, i.e., the same average length). According to Carman-Kozeny, this would mean that the random network should become more permeable than the hexagonal one as channels are gradually closed! This result might look surprising. However, one should keep in mind that the comparison being made at fixed $g_{s}$, wider liquid channels also remain in the random network. Since Carman-Kozeny predicts that the permeability $\mathrm{K}\left(\mathrm{g}_{\mathrm{s}}\right)$ multiplied by $\left(\mathrm{S}_{\mathrm{s}}\left(\mathrm{g}_{\mathrm{s}}\right)\right)^{2}$ should be a function of $\mathrm{g}_{\mathrm{s}}$ only,[17] this assumption can be tested using the KPL model.

Taking again a small volume element of uniform temperature, the flow across the network was calculated with the following boundary conditions: imposed pressures, $\mathrm{p}_{1}$ and $\mathrm{p}_{2}$, on the vertical boundaries and no flow on the horizontal ones. The specific masses of the solid and liquid phases were assumed to be equal and constant, so that the KPL model resumed in this case to a Kirchhoff-Poiseuille calculation without losses. The permeability calculated for the hexagonal and random networks of grains is shown in Fig. 6 for $0.9<g_{s}<1$. As can be seen, the random network of grains is slightly more permeable than the hexagonal one up to very high volume fraction of solid (0.995). At such high value of $g_{s}$, the granular (or hexagonal) model is meaningless as alloys exhibit isolated pockets of liquid rather than continuous films. It should be noted that the calculation for the random network of grains is stopped at a lower value of $g_{s}$. This is due to the fact that it becomes difficult to find a liquid path across the calculation domain when $g_{s}$ is close to unity, unlike the hexagonal network which exhibits a uniform channel width throughout this domain at any value of $g_{s}$. At lower volume fractions of solid, the higher permeability of the random network of grains is rather surprising as its associated solid-liquid interfacial area was larger than that of the hexagonal network (Fig. 5). Therefore, at least one of the two models does not follow Carman-Kozeny's relationship.

In order to check this point, the permeability $\mathrm{K}$ shown in Fig. 6 was normalised with the square of the effective interfacial area, $\mathrm{S}_{\mathrm{V}}{ }^{2}$ (see Fig. 5). The result shown in Figs 7 and 8 demonstrate that the value of $\mathrm{KS}_{\mathrm{V}}{ }^{2}$ for the random network of grains follows very closely the 
Carman-Kozeny relationship $\left((1-g s)^{3} /\left(5 g_{s}{ }^{2}\right)\right.$, Eq. 13) up to very high volume fraction of solid. For gs close to unity (Fig. 8), the random network of grains deviates from this relationship which then becomes closer to the values obtained for the hexagonal network. It is interesting to note that this transition occurs at a value of $g_{s}$ larger than that associated with the decrease of the solid-liquid interfacial area, $\mathrm{S}_{\mathrm{sl}}$.

In summary, the transition seen in the effective solid-liquid interfacial area at $g_{s}=0.95$ for the random network of grains corresponds to a disappearance of the smallest liquid channels which overcomes the natural increase of $\mathrm{S}_{\mathrm{sl}}$ in $\sqrt{\mathrm{g}_{\mathrm{s}}}$ when all the channels are open. However, at this value, the channels remain largely interconnected (see the enlargement micrograph in the middle of the mushy zone of Fig. 4). A second transition occurs in the Voronoi network when the permeability no longer follows the Carman-Kozeny relationship (see Fig. 8). This would correspond to the formation of isolated liquid pockets which are still accounted for in the calculation of $g_{s}$, but do not play anymore a role in the determination of $K$ [4]. In the present case, liquid pockets are in fact represented by isolated liquid films. Considering a smooth rather than a piece-wise linear solid-liquid interface for the grains would certainly decrease the value of $g_{s}$ at which this second transition occurs. On the opposite, considering a 3D situation, the intergranular liquid will be able to by-pass a solid bridge formed between two grains, while it is stopped in 2D.

A major interest of the KPL model for the prediction of hot tearing formation is really in its capability to evidence localisation of feeding. A calculation was performed for a mushy zone directionally solidified in a thermal gradient of $10^{3} \mathrm{~K} / \mathrm{m}$ (i.e., 10 times smaller than that used in Fig. 4 to see the grains) with a velocity of $10^{-3} \mathrm{~m} / \mathrm{s}$ (i.e., same cooling rate). The other conditions of this KPL simulation were: density of the solid, $\rho_{\mathrm{s}}=2620 \mathrm{~kg} / \mathrm{m}^{3}$, density of the liquid, $\rho_{\mathrm{l}}=2440 \mathrm{~kg} / \mathrm{m}^{3}$, viscosity, $\mu=1.5 \cdot 10^{-3}$ Pa s. As shown in Fig. 9 for a small portion of the random network of grains, the intergranular liquid pressure within the channels depends very much on the local configuration of the grains. In this figure, the pressure is represented with various grey levels, the scale of which being shown on the right. For visualisation purpose, the grey levels are indicated for each polyhedron associated with the triple junctions of the grains, even though it has a meaning in the channels only. As can be seen, some regions of the grain structure are no longer fed (white areas). Region 1 (light grey) has an intergranular pressure only 100 Pa below the imposed boundary value (see Fig. 3). While in 
the upper part of Fig. 9 the pressure gradually decreases as one goes to lower temperatures on the right (regions labelled 2, 3, 4), in the bottom part it makes an abrupt jump from -100 $\mathrm{Pa}$ (region 1) to -82 kPa (region 7) within two single channels. These channels are indeed fairly narrow and are the only path of liquid to a large cluster of grains where feeding is still necessary (dark region where the depression is on the order of $1 \mathrm{MPa}$ ). Most likely, such regions will be subject to porosity formation in order to relax these large depressions. This localisation of feeding is one of the key parameter, besides localisation of strains, to the hot tearing problem.

\section{Conclusion}

Although the KPL model is still two dimensional and has some drastic assumptions (in particular piecewise linear solid-liquid interfaces with no exchange of solute between the various domains), it already brings some valuable information. First, it has shown that the global permeability of a random network of grains follows fairly accurately the CarmanKozeny relationship. Second, it is capable of showing a localization of intergranular liquid feeding, despite the global correspondence with Carman-Kozeny's relationship. The two transitions evidenced by the present model, i.e., decrease of $S_{s l}$ beyond $g_{s}=.95$ and deviation from Carman-Kozeny's relationship at $g_{s}$ close to unity, do not occur with a regular arrangement of grains. Third, taking into account some coalescence criterion, it can predict the formation of clusters of grains and thus the gradual transition from a network of continuous liquid films to a continuous network of interconnected solid grains (i.e., percolation of grains). Fourth, it provides an excellent framework for future developments. The computation costs being fairly small, the model can be extended in several ways. These developments will encompass an extension to 3 dimensions and the account of solid contraction. This last point is of crucial importance for hot tearing. As demonstrated by experiments, it is well known that the sensitivity of alloys to hot cracking increases with the grain size. Therefore, despite having small grains in a mushy zone, the clustering of grains increases the size of the zones which become coherent from the point of view of thermal contraction. When the grains are isolated, thermal contraction simply adds to solidification shrinkage at the boundary of each grain. When they are clustered, it is concentrated at the external boundary of the entire cluster, thus enhancing drastically the localization of feeding. 
One of the future developments will be to consider thermal shrinkage at the level of the grains/clusters.

\section{Acknowledgements}

The authors would like to acknowledge the financial support of Alcan-Pechiney, Research Center of Voreppe (CRV), France. They would like to thank also Ph. Jarry, from the CRVAlcan at Voreppe, and V. Mathier for very fruitful discussions.

\section{References}

1. D.G. Eskin, Suyitno, L. Katgerman, Progr. Materials Science 49 (2004) 629-711.

2. I. Farup, J.-M. Drezet, M. Rappaz, Acta Mater. 49 (2001) 1261-69.

3. M. Rappaz, J.-M. Drezet, M. Gremaud, Met. Mater. Trans. 30A (1999) 449-55.

4. M. Braccini, Ch. Martin, M. Suéry, Y. Bréchet, in: Modeling of Casting, Welding and Advanced Solidification Processes IX, P. R. Sahm, P. N. Hansen, J. G. Conley Eds (Shaker Verlag, Aachen, Germany, 2000) p. 18.

5. M. M’Hamdi, A. Mo, Ch.. Martin, Met. Mater. Trans. 33A (2002) 2081-93.

6. M. M’Hamdi, A. Mo, H. G. Fjaer, submitted to Met. Mater. Trans. (2004).

7. M. Rappaz, A. Jacot, W. Boettinger, Met. Mater. Trans. 34A (2003) 467-479.

8. V.Mathier, A. Jacot, M. Rappaz, Modelling Simul. Mater. Sci. Eng. 12 (2004) 479-90.

9. W. O. Dijkstra, C. Vuik, A. J. Dammers, L. Katgermann, in Solidification Processes and Microstructures, M. Rappaz, Ch.Beckermann, R. Trivedi Eds (TMS Publ., Warrendale, PA, USA, 2004) 151-6.

10. A. Karma, private communication.

11. Ch. Charbon, M. Rappaz, Acta Mater. 44 (1996) 2663-8.

12. C. Bradford Barber, D. P. Dobkin, H. Huhdanpaa, ACM Trans. Math. Soft.. 22 - 4 (1996) 469-83.

13. X. Doré, H. Combeau, M. Rappaz, Acta Mater. 48 (2000) 3951-62.

14. I. Ohnaka, Trans. ISIJ 26 (1984) 1045.

15. S. Vernede, M. Rappaz, to be published.

16. A. Jacot, M. Rappaz, Acta Mater. 50 (2002) 1909-26

17. O. Nielsen, L. Anberg, A.Mo, H. Thevik, Met. Mater. Trans. 30A (1999) 2455-62. 


\section{Figure captions}

Figure 1 : Schematics of the granular model of microsegregation used for the calculation of solidification of a random family of globular grains.

Figure 2: Schematics of the Poiseuille flow within half a channel, including the liquid feeding of the moving solid-liquid interface, and of the Kirchhoff law at one triple junction.

Figure 3 : Schematics of the overall liquid feeding within a regular network of hexagonal grains, with associated boundary conditions.

Figure 4: Calculated mushy zone of an alloy solidifying with a globular structure in a strong thermal gradient $(100 \mathrm{~K} / \mathrm{cm})$ at $1 \mathrm{~K} / \mathrm{s}$. The enlargements on the left come from the rectangles indicated on the overall grain structure, while the curve on the right presents the volume fraction of solid calculated in horizontal sections.

Figure 5 : $\quad$ Effective solid-liquid interfacial area, $\mathrm{S}_{\mathrm{s}}$, as a function of the volume fraction of solid, as calculated from the granular model for the hexagonal and random grain networks. The effective solid-liquid interface has been normalised by the average grain size, $\left(\mathrm{V}_{\text {tot }} / \mathrm{N}\right)^{1 / 2}$ where $\mathrm{V}_{\text {tot }}$ is the volume (surface) of the domain and $\mathrm{N}$ is the number of grains. The theoretical curve calculated for the hexagonal network is also shown.

Figure 6 : Permeability, $\mathrm{K}$, as a function of the volume fraction of solid, $\mathrm{g}_{\mathrm{s}}$, for the hexagonal and random networks of grains, as calculated with the PKL model.

Figure 7: Permeability, $\mathrm{K}$, normalised with the square of the intrinsic effective solidliquid interface, $\mathrm{S}_{\mathrm{v}}$, as calculated for the hexagonal and random networks of grains with the PKL model. The Carman-Kozeny relationship is also represented for comparison.

Figure 8 : $\quad$ Enlargement of Figure 7 for very high fraction of solid.

Figure 9 : $\quad$ Pressure map for a small region of a random network of grains as calculated with the PKL model. Although grey levels are indicated for the surface, the pressure has a meaning within the intergranular liquid channels only. White areas correspond to channels which are no longer fed. 


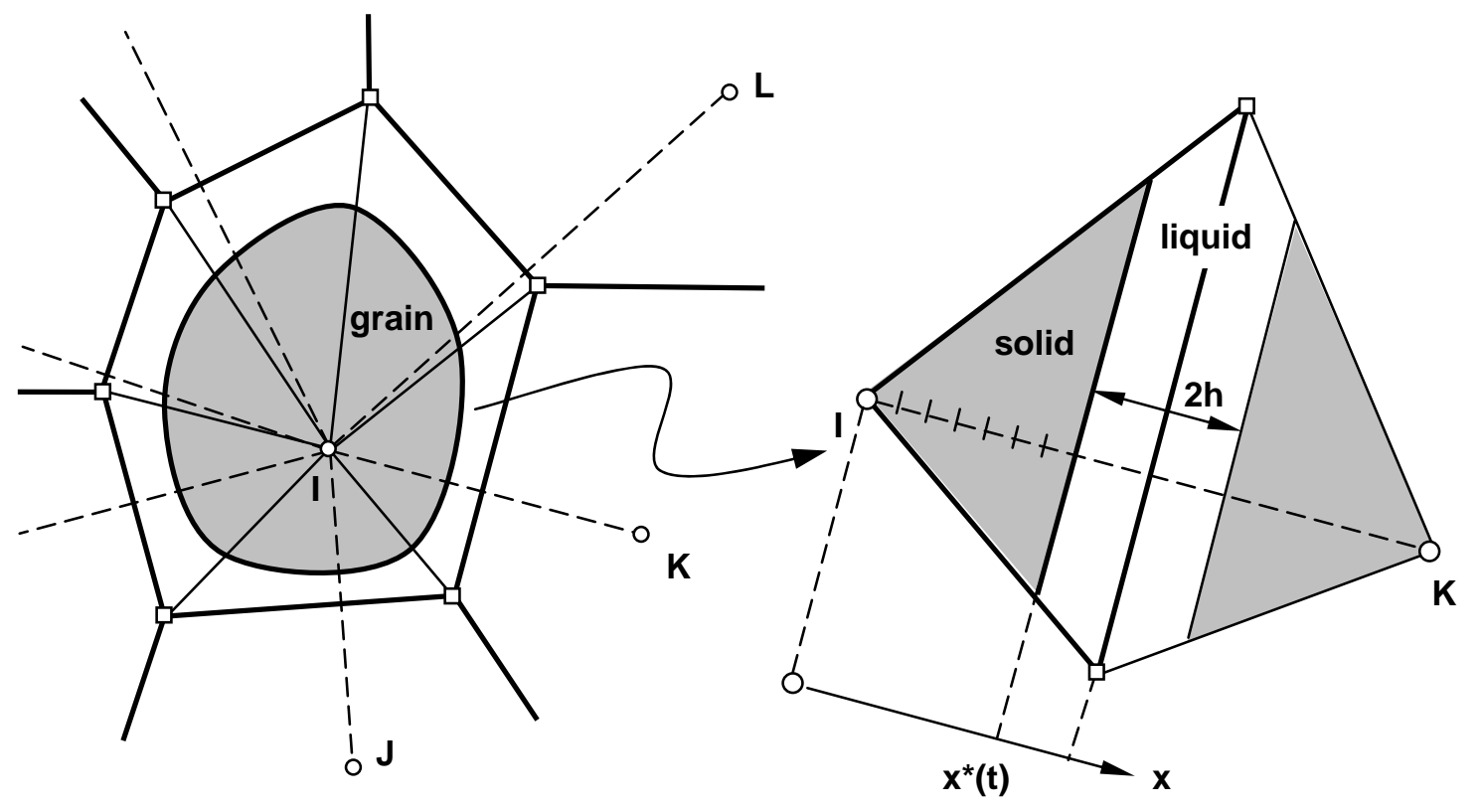

(a)

(b)

Figure 1 


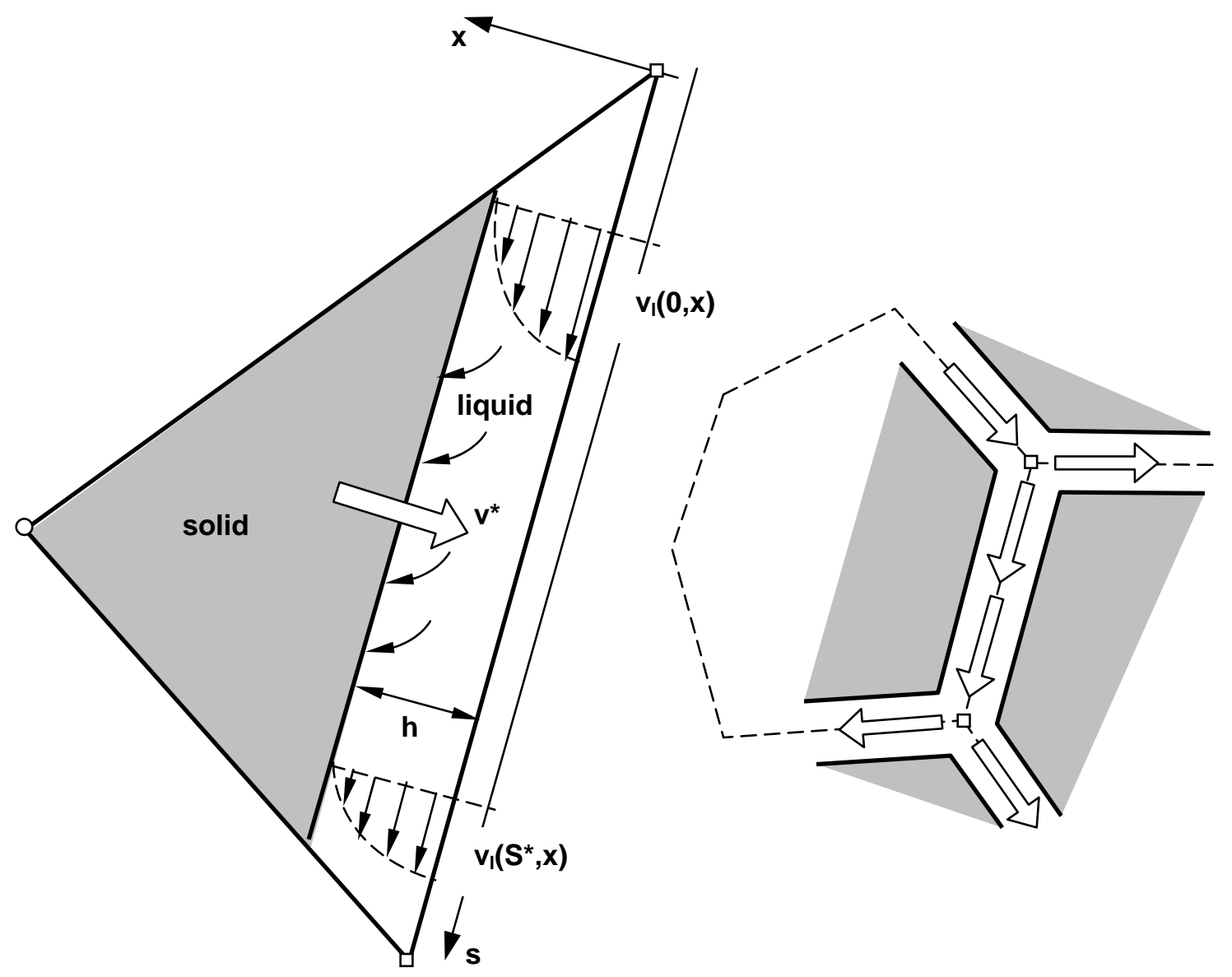

(a)

(b)

Figure 2 


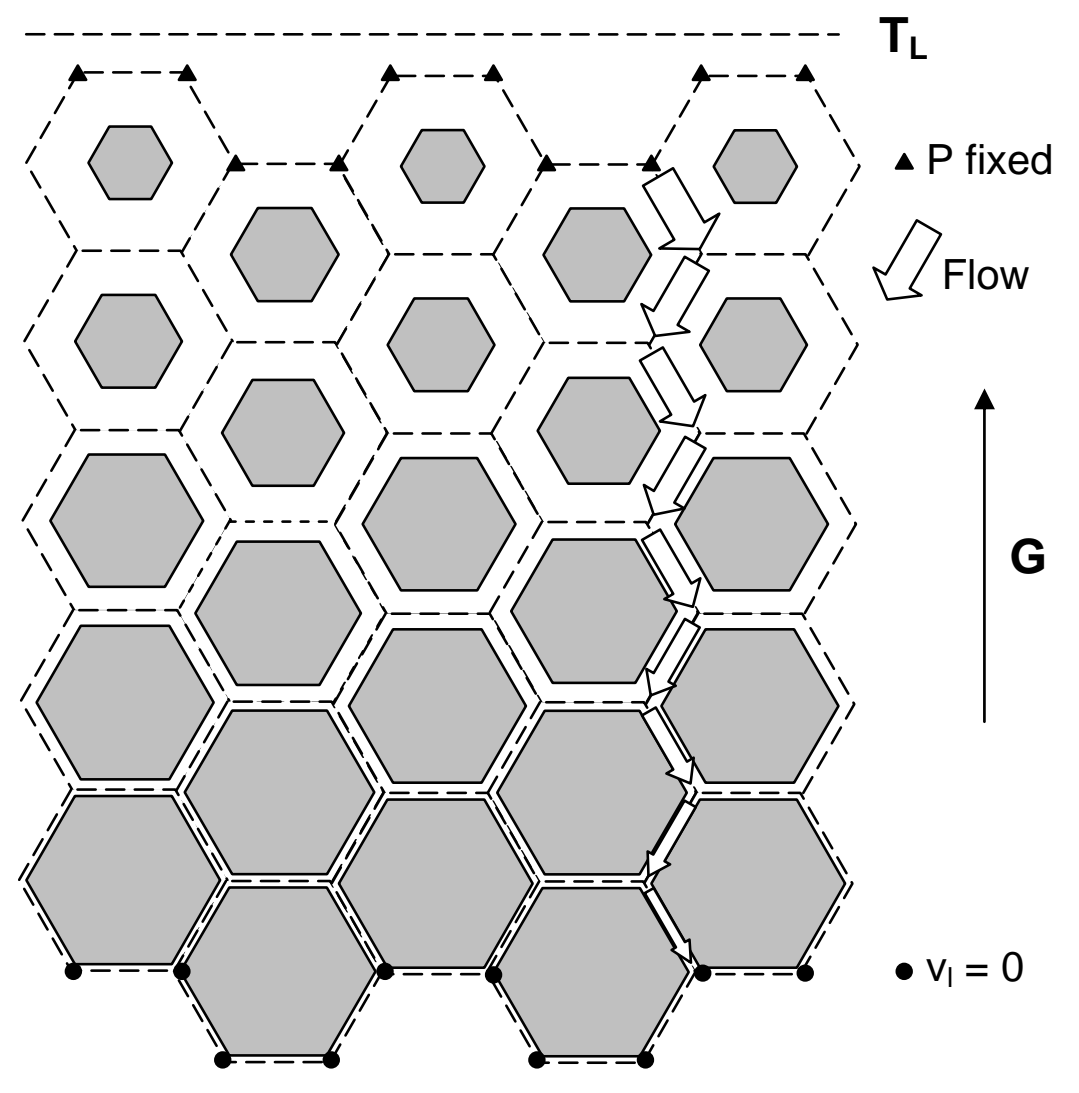

Figure 3 

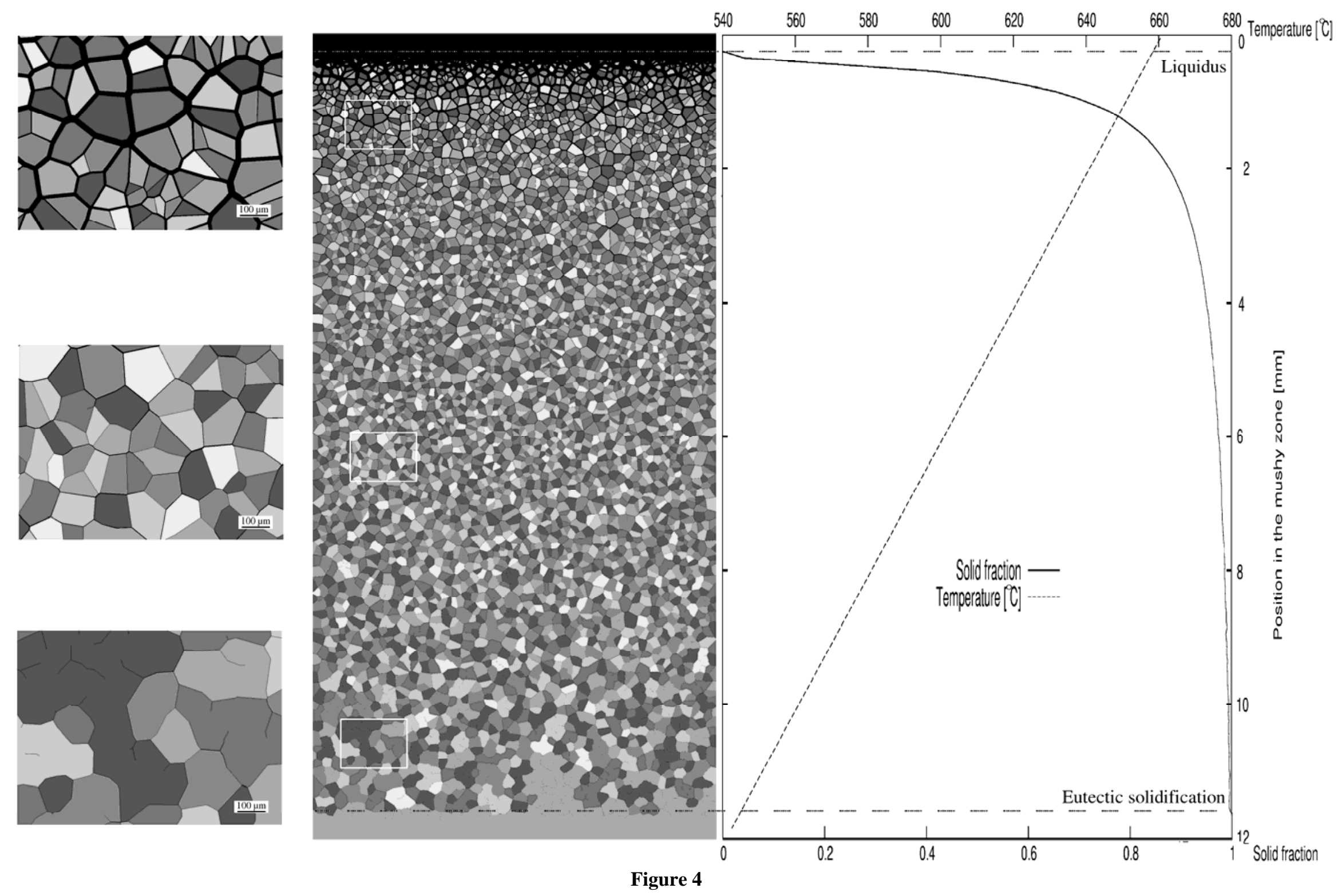


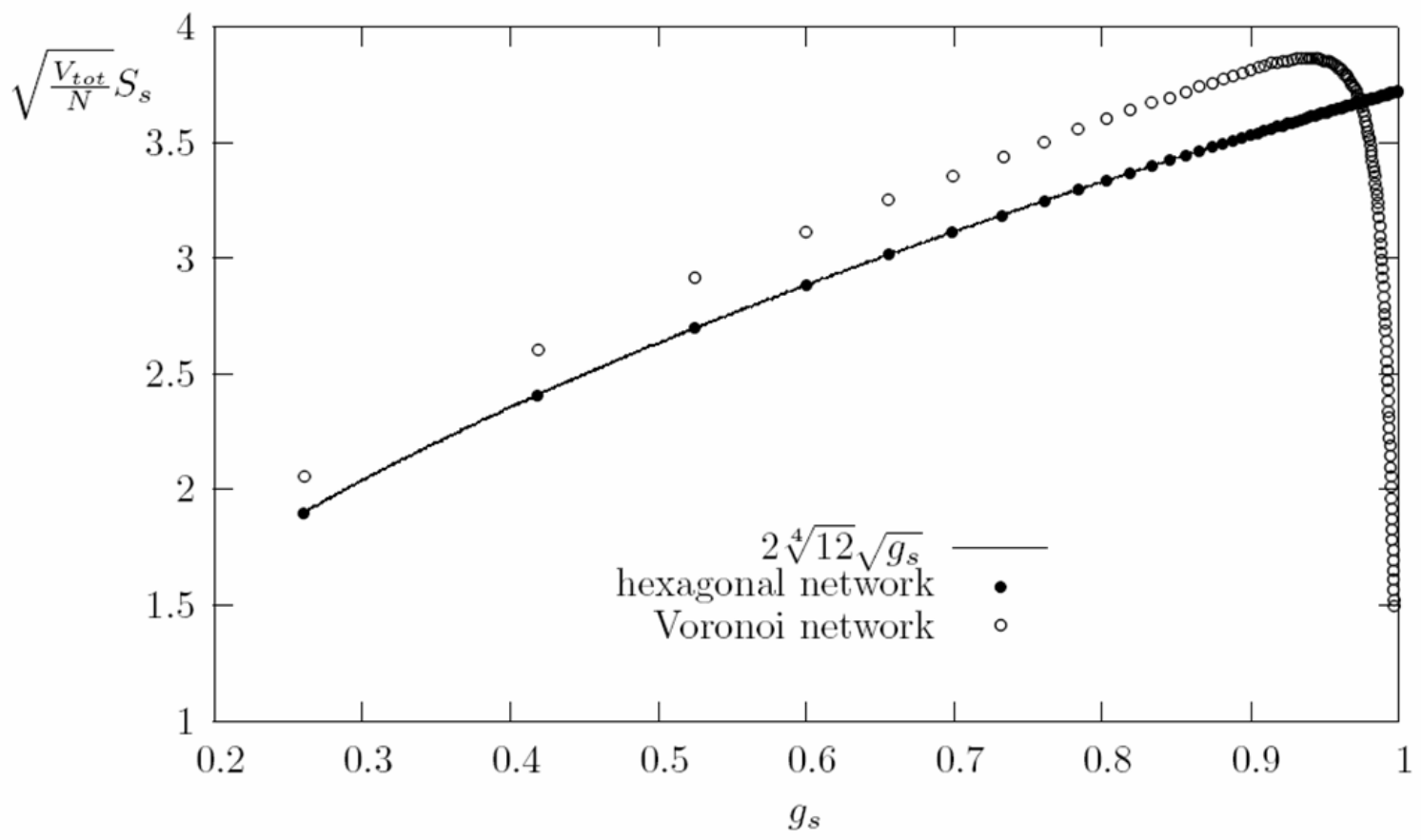

Figure 5 


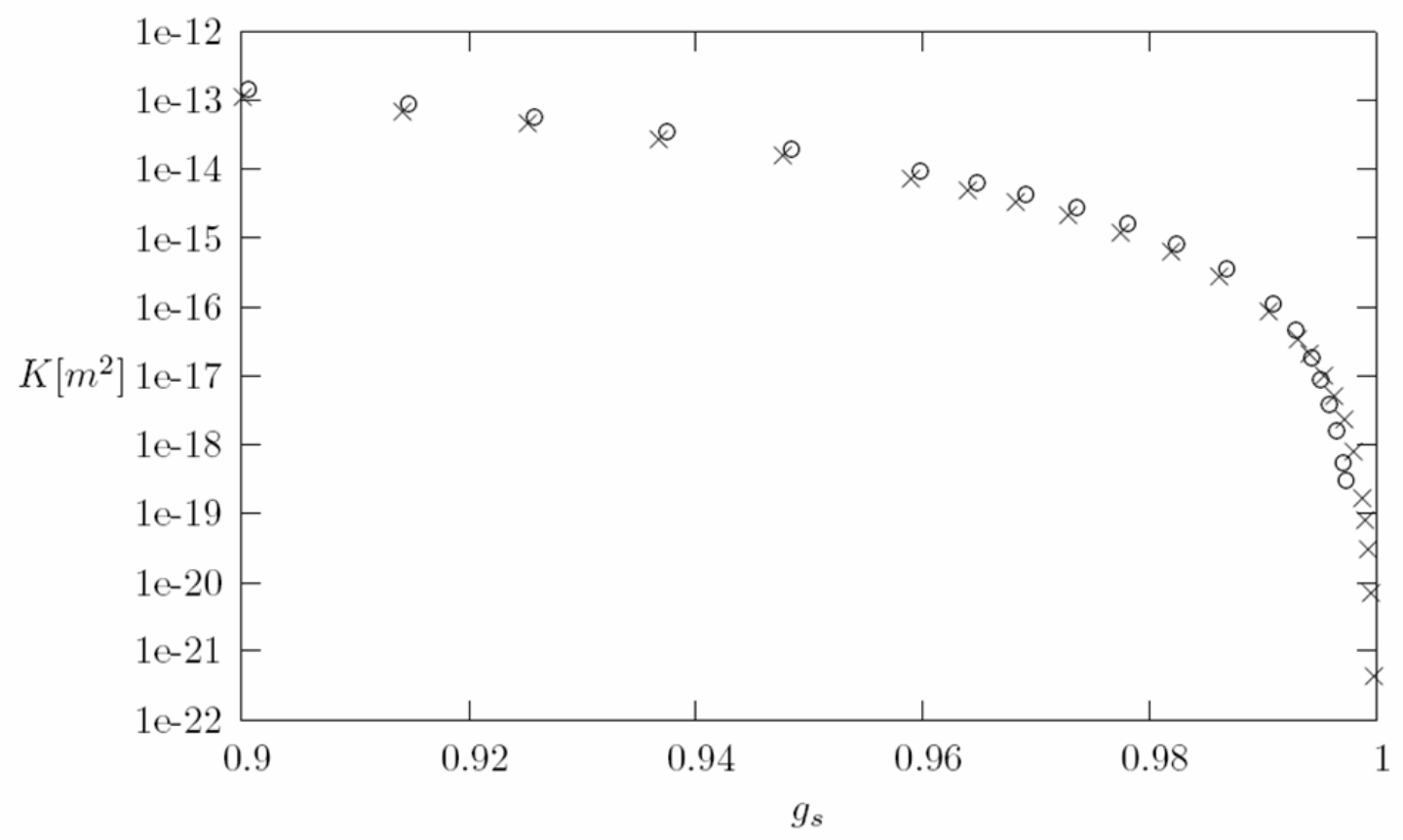

Figure 6 


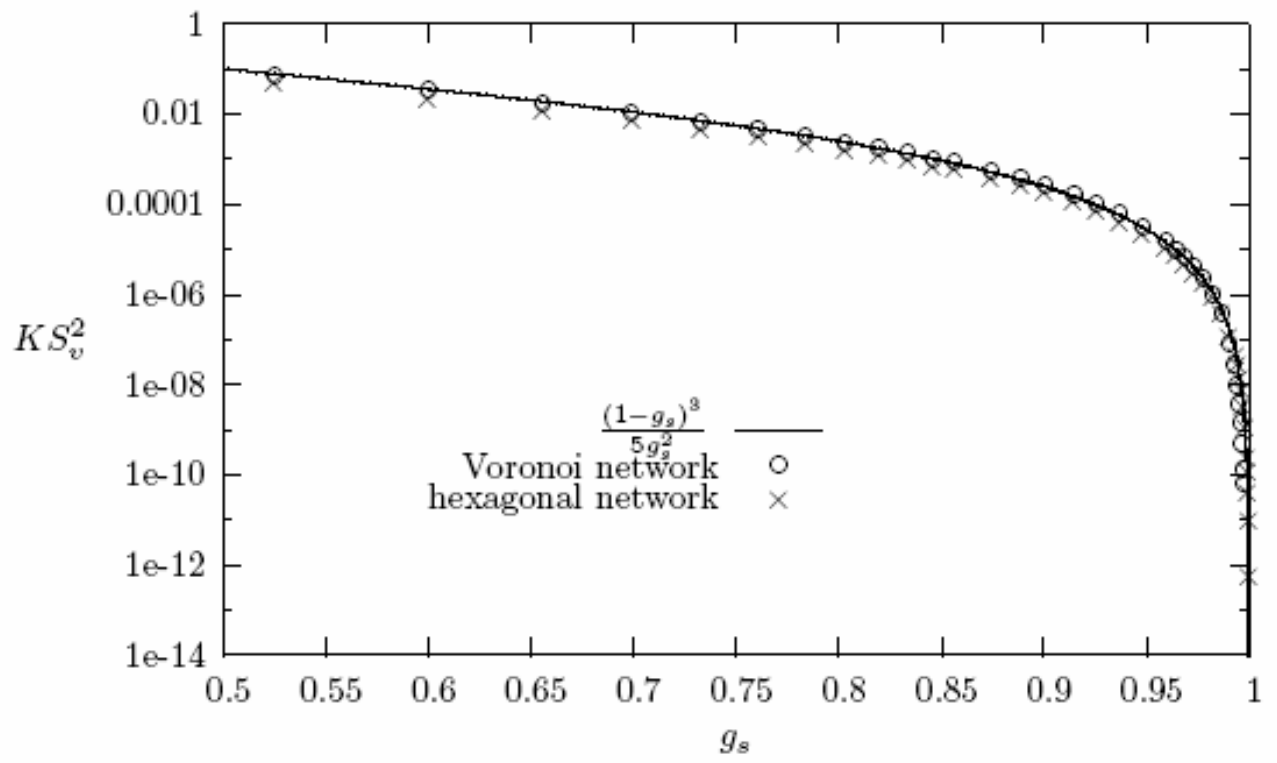

Figure 7 


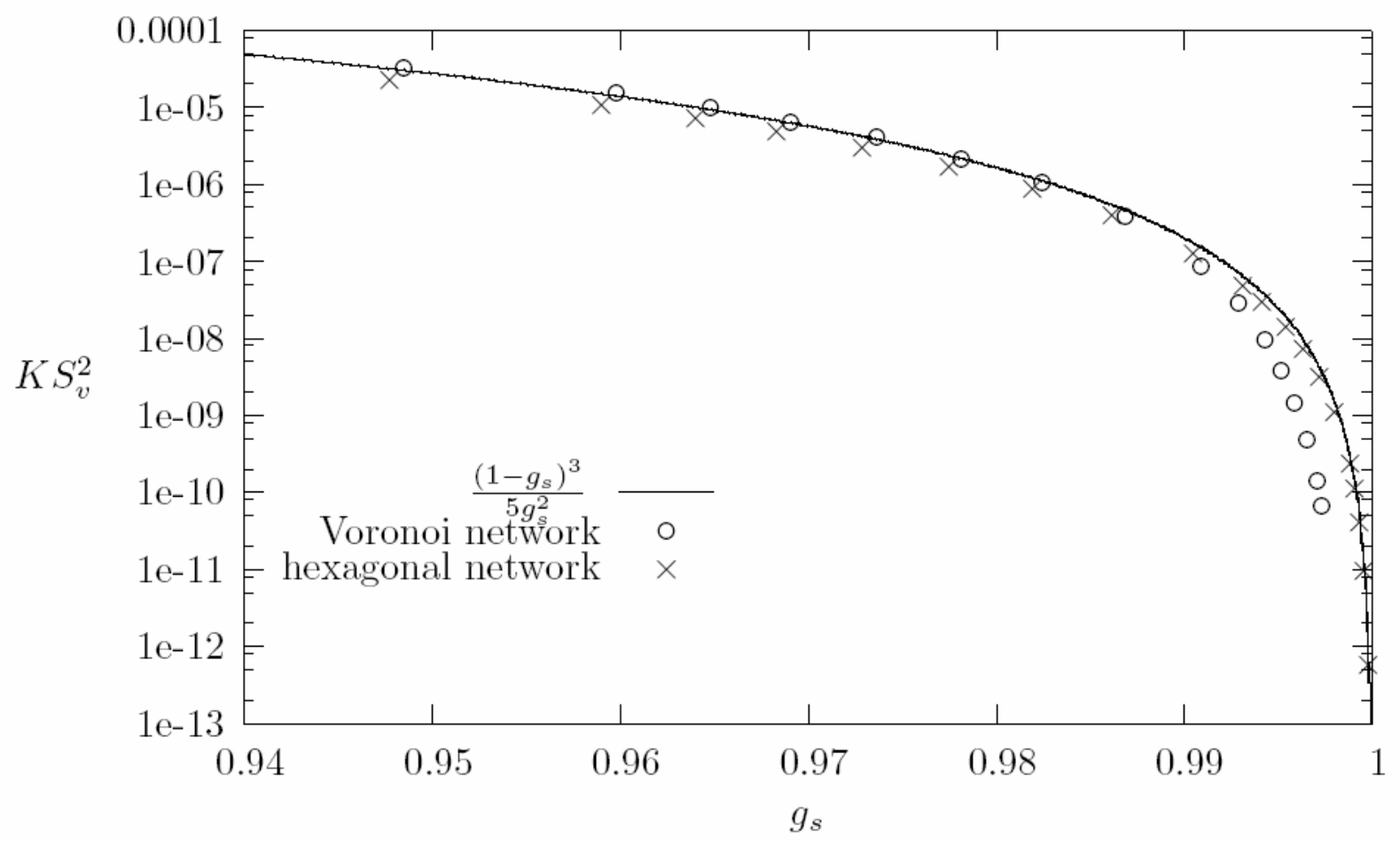

Figure 8 


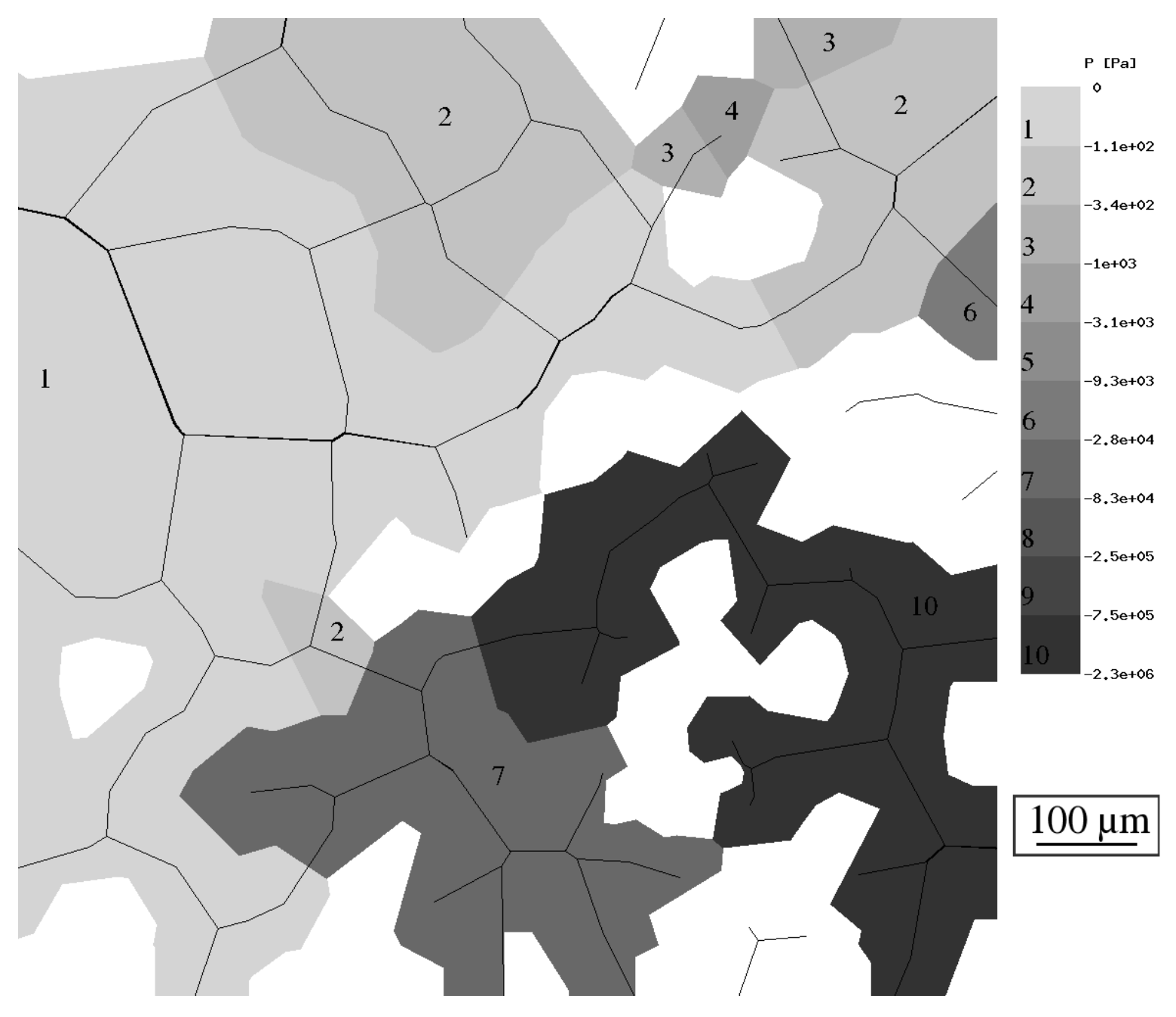

Figure 9 